\title{
Tesis doctorales en España: análisis de la bibliografía específica y su accesibilidad
}

PhD thesis in Spain: an analysis of the specific bibliography and access

\section{Llorenç ARguimbau I Vivó (1) y M. Eulàlia Fuentes I Pujol (2)}

(1) Observatori de la Recerca. Institut d'Estudis Catalans. 08001 Barcelona. Correo-e: llarguimbau@iec.cat. - Àrea de Documentació. Universitat Autònoma de Barcelona. 08193 Bellaterra. Correo-e: Ilorenc.arguimbau@uab.cat. (2) Àrea de Documentació. Universitat Autònoma de Barcelona. 08193 Bellaterra. Correo-e: eulalia.fuentes@uab.cat

\begin{abstract}
Resumen
A raíz de un artículo presentado a revisión para ser publicado, titulado "Las tesis doctorales en España (1998-2008): Estudios, estadísticas y repositorios cooperativos", realizamos un trabajo previo de recopilación, lectura y análisis de toda la bibliografía específica relevante sobre el tema. En este artículo se pretende dar a conocer esta documentación, analizándola individualmente y en conjunto, para ver si nos permite radiografiar los usos y aplicaciones de las tesis doctorales, pero también su difusión, evaluación y las formas de acceso y consulta.
\end{abstract}

Palabras clave: Tesis doctorales. Radiografía. Universidad española. Accesibilidad. Consulta. Difusión

\section{Introducción}

Como se ha expuesto en el resumen, a raíz del trabajo previo realizado para escribir un artículo sobre las tesis doctorales en España (19982008), que requirió la revisión bibliográfica exhaustiva de la literatura sobre el tema, creímos conveniente que todo este trabajo, muy laborioso e interesante, pudiese también ser compartido, hacerlo público, y por ello decidimos presentarlo como ponencia en el marco del congreso IBERSID 2009, XIV Encuentros Internacionales sobre sistemas de Información y Documentación ,y fue aceptada.

Por lo tanto, la ponencia tiene como objetivo dar a conocer la bibliografía analizada, su utilidad, las reflexiones de sus autores y, si es posible, establecer una radiografía sobre su acceso, teniendo siempre presentes los factores de transformación de los últimos años: adaptación al Espacio Europeo de Educación Superior (EEES), y por lo tanto la existencia de una nueva normativa, y el impacto de las tecnologías de la Información y la Documentación.

La revisión bibliográfica de la literatura profesional sobre el tema objeto de análisis se ha reali-

\begin{abstract}
A bibliographical review of the literature on PhD thesis in Spain is presented. Their uses and applications, assessment, dissemination, access and reading are evaluated.
\end{abstract}

Keywords: PhD thesis. Assessment. Accessibility. Dissemination. University. Spain.

zado consultando las fuentes de información que se enumeran a continuación:

- Base de datos bibliográfica del Centro Superior de Investigaciones Científicas (CSIC),

- E-PRINTS in Library and Information Science (E-LIS),

- ISI Web of Knowledge,

- Library Information Science \& Technology Abstracts (LISTA),

- TESEO, Base de datos de Tesis Doctorales, y

- TROBADOR, metabuscador de recursos electrónicos de las bibliotecas de la Universitat Autònoma de Barcelona (UAB).

En ellas se han recuperado fundamentalmente artículos de revistas, ponencias y comunicaciones a congresos, con perspectivas generales y especializadas.

\section{Usos y aplicaciones de las tesis doctorales}

Las tesis doctorales se pueden analizar desde distintos puntos de vista. Son los trabajos de investigación por excelencia, son instrumentos 
para alcanzar un nuevo grado académico, son indicativos de la producción científica y son instrumentos de colaboración entre las universidades y las empresas. A continuación se irán desarrollando cada uno de estos puntos de vista a partir de una selección de las opiniones de los distintos autores recogidas de la revisión bibliográfíca exhaustiva realizada sobre el tema.

\subsection{Tesis doctorales como trabajos} de investigación $\left(^{*}\right)$

La tesis doctoral es un trabajo de investigación riguroso, el trabajo por excelencia, (...). Tiene que ser un trabajo inédito, de creación y por tanto requiere que previamente se analice y estudie todo lo que se haya escrito, investigado y trabajado sobre el tema escogido. (Fuentes, 1992, p. 21)

Las tesis doctorales, desde la perspectiva de la investigación, son buenos documentos para caracterizar la investigación original en un campo. (...) La tesis doctoral es el primer trabajo científico, aquél que abre las puertas al mundo de la investigación, y seguramente, uno de los que más esfuerzos y recursos (sobre todo humanos y de tiempo) consume en la vida de un investigador. Pero, ante todo es, o debería ser, un trabajo de investigación original, esto es, debe aportar nuevo conocimiento. $Y$ para acreditarlo se establece un sistema de control y de evaluación mediante un tribunal de personas, sabias en la materia, que lo certifica. (Delgado et al., 2006, p. 495)

No se debe olvidar que una de las misiones básicas de la universidad es la formación de investigadores y que la aprobación de la tesis doctoral supone la suficiencia investigadora.

Las tesis doctorales son trabajos de investigación científica que además tienen que reunir todos los requisitos formales exigidos por la investigación científica, puesto que son expuestas en público y necesitan la aprobación de un tribunal, y también de todos los conceptos básicos en los que se apoya la investigación científica, la ciencia y el método científico. (Fuentes y González, 2002, p. 231)

La tesis doctoral es la concreción de dos importantes funciones de la universidad: la investigación y la formación de nuevas generaciones. Mediante la tesis doctoral el alumno adquiere las competencias que supone el grado superior de los estudios universitarios, y, a la vez, la iniciación en el mundo de la investigación. (Miguel, 2000, p. 645 ss)

Este tipo de documento científico [las tesis doctorales] forma parte del inicio de la producción científica. Partiendo del supuesto de que la tesis doctoral es un trabajo académico de iniciación científica, marco inicial de la especialidad de un investigador, constituyendo una fuente de información que refleja el logro en su propio campo del saber, se considera de gran importancia la recopilación y su análisis, para el estudio de una determinada producción científica. (Muñoz, 2004, p. 499)

Una de las misiones básicas de la universidad es la formación de sus alumnos en la investigación científica. Esta misión debe alcanzar su máxima expresión en los estudios de doctorado, en cuanto su realización acredita académicamente la plena capacidad investigadora. Precisamente por ello, la tesis doctoral debe constituir un trabajo de investigación científica. (...). Según su naturaleza sustantiva, la tesis doctoral no ha de ser otra cosa, pues, que una investigación científica. Es más, si no en el fondo, es decir, en la importancia de los descubrimientos, en todo lo demás se la puede considerar como el prototipo de los trabajos de investigación. (Sierra Bravo, 1994, p. 23)

\subsection{Tesis doctorales como instrumentos para alcanzar un nuevo grado académico}

La tesis doctoral es un trabajo de investigación realizado para obtener el Grado de Doctor, que lleva a cabo el doctorando en un departamento universitario o centro de investigación, bajo la orientación de un director de tesis. (Miguel, 2000, p. 645)

La tesis doctoral es el primer trabajo de investigación en el sentido más estricto, por lo que debería ser el primer trabajo publicado en el tiempo. Su aprobación supone la suficiencia como investigador. Su objeto es suficientemente amplio de acuerdo con las características de este tipo de trabajo. Permite obtener el Título de Doctor. (López Yepes, 1995, p. 23)

La tesis doctoral es un trabajo de investigación riguroso, (...), que se requiere para obtener el grado de Doctor, después de la realización del tercer ciclo y por lo tanto es el prólogo de la especialización posterior de un investigador. Es un trabajo de investigación importante, que ha de ser juzgado por un tribunal de doctores especialistas en el tema y dirigido por uno de ellos. (Fuentes, 1992, p. 21)

Las motivaciones que conducen a una persona a realizar el doctorado [son muy distintas], puesto que no será la misma motivación la de alguien que aspira alcanzar una plaza en docencia que la de otra persona cuyo interés es alcanzar un mayor grado académico o la de un 
tercero que pretende profundizar en determinada área del conocimiento o perfeccionar su desempeño en la investigación. (Agudelo et al., 2003, p. 607)

La defensa de tesis doctorales, en el caso del profesorado, va unida a la carrera académica y a la formación de los mismos, pues es requisito sine qua non para obtener, mediante oposición, la titularidad. (Muñoz, 2004, p. 499)

Una tesis doctoral supone la culminación del ciclo de aprendizaje e investigación de un científico en formación bajo la dirección de uno o varios doctores. Una vez superada la defensa pública de la investigación ante un tribunal de expertos en la materia, el doctorando obtiene el título de doctor en su especialidad y, a partir de ese momento, tiene la posibilidad de difundir el conocimiento generado a través de la publicación de la tesis o de otros documentos (artículos, comunicaciones, etc.) (Fuentes; Arguimbau, 2009)

\subsection{Tesis doctorales como indicativos} de la investigación y producción científica (Ver anexo II)

La investigación científica es la actividad encaminada a la obtención de conocimiento científico- esencialmente humana que tiene como blanco principal la búsqueda de la verdad, la extensión de los conocimientos en el investigador y en su trascendencia a los demás. Verdad y comunicación son, pues, los dos pilares en que se asienta el proceso de investigación científica. (López Yepes, 1995, p. 40)

La tesis doctorales, juntamente con las monografías y las publicaciones periódicas o las reuniones científicas (conferencias, jornadas, seminarios, reuniones, (...) permiten esbozar una panorámica descriptiva de la literatura científica en general, pero también en campos concretos, consolidándolos. (Fuentes-González, 2001, p. $25,35)$

[Las tesis doctorales], es claro que constituyen un indicativo de gran valor de la producción científica y no un requisito [solo] para obtener un título académico. Además, cabe valorar la dirección de tesis como investigación y como docencia. (Agudelo et al., 2003, p. 595)

Para valorar el nivel de consolidación de una disciplina científica se pueden observar y valorar diversas variables y una de ellas son las tesis doctorales, trabajos de investigación en el ámbito académico. (...). Dado que las tesis doctorales tienen que tratar siempre un tema novedoso y original, o bien una nueva revisión o estudio y si a eso le sumamos que además constituyen el trabajo de investigación por excelencia, la revisión de ellas, muestra el nivel de consolidación de una disciplina científica, y permite además, plasmar los cambios producidos en ella y escribir su historia. (Fuentes y González, 2002, p. 231-232)

De la identidad de naturaleza entre la tesis doctoral y la investigación científica, se derivan dos consecuencias de interés, (...). Según la primera, de carácter terminológico, lo que se diga de la investigación científica es extensible a la tesis doctoral y viceversa, dejando a salvo a los aspectos académicos peculiares de la tesis. Por ello, con dicha salvedad, ambos términos se utilizaran aquí como sinónimos. De acuerdo con la segunda, los conceptos básicos en que se apoya la investigación científica, la ciencia y el método científico, también serán válidos como tales en el caso de la tesis doctoral. (Sierra Bravo, 1994, p. 23).

Por consiguiente, las tesis doctorales son buenos espejos en que se reflejan las líneas y tendencias científicas de la Universidad. Pero, en la medida en que para su elaboración requieren de una relación maestro/discípulo (...), y para su aprobación exigen el refrendo de la academia universitaria a través de un tribunal formado al efecto, las tesis son un buen medio para conocer tanto los focos de generación de investigación como las escuelas científicas en que se concretan. En definitiva, son un excelente referente para conocer la estructura social de la investigación en la universidad. (Delgado et al., 2006, p. 495)

Según López Yepes, una de las fuentes documentales más importantes para estudiar el estado de la investigación de un país, a través de su literatura científica, es la producción correspondiente a las tesis doctorales. La tesis doctoral, en cuanto está sometida al escrutinio y examen de un tribunal, parece obligado en todo caso que reúna del modo más completo posible los requisitos formales exigidos por la investigación científica. (Fernández et al., 2003, p. 163)

\subsection{Tesis doctorales, instrumentos} de colaboración entre las universidades y las empresas

La universidad debe intentar ser cada vez más competitiva y responder a esta competitividad mediante un incremento de la calidad, tanto a nivel docente como investigador. Tiene que ayudar a las empresas proporcionándoles personal capaz, bien formado, que sepa documentarse e informarse correctamente, que sepa dónde buscar la información y como valorarla, que se adapte a las necesidades cambiantes de 
la llamada sociedad de la información. Pero a su vez, la empresa necesita realizar investigaciones, obtener resultados que puedan aplicarse; y ello lo puede conseguir bien con investigadores propios, o bien contratando, becando o mediante convenios de colaboración con los núcleos docentes, principalmente con las universidades y los institutos de investigación. Pero no solo las universidades y las empresas deben colaborar estrechamente, sino que los gobiernos tienen que elaborar políticas de información y documentación y de investigación. (FuentesGonzález, 2001, p. 25, 35).

Las empresas y el gobierno están en deuda con la universidad. No puede mantenerse un nivel alto de investigación y por ende de saberes propios, sin una cooperación plena de los tres: universidades, empresas y gobierno. La reingenieria del entorno investigador debe pasar, desde gobierno y empresas, por un incremento en la productividad investigadora de la universidad y esto es cooperación altruista, pero con un legítimo interés en los resultados, que permitirán la auténtica competitividad. (Fluxá, 1996, p. 899)

Que la investigación sea replicable, es decir, que pueda ser sometida a revisión e incluso reproducción en su planteamiento, desarrollo y ejecución. (Sierra Bravo, 1994, p. 49)

Ciertamente, la vinculación o binomio ciencia/sociedad es cada vez mayor debido a que: los descubrimientos científicos se acercan cada vez más a su aplicación práctica en la sociedad; el científico es, a la vez, investigador y formador de investigadores; los contactos entre los poderes públicos y los científicos son cada vez mayores a pesar de la desconfianza mutua. Se observa que la ciencia y las cuestiones a ella referidas -docencia e investigación- se convierten con frecuencia en debate político. (López Yepes, 1995, p. 45)

\section{Acceso, evaluación y difusión de las tesis doctorales}

El acceso a las tesis doctorales no siempre resulta fácil, no cuenta con una normativa de carácter general y en todo caso no se rige por criterios uniformes (Moralejo, 2000, p. 235).

De los datos expuestos pueden deducirse las siguientes conclusiones (Moralejo, 2000, p. 242243):

1. No puede dejar de sorprender que, pese a la importancia de las tesis doctorales, tanto como resultado de la actividad investigadora de la universidad como por el volumen que han alcanzado en la actualidad, no exista una normativa de carácter general y sean tan escasas las de carácter interno, que favorezca una gestión uniforme de las tesis, garantice su conservación, difusión y acceso y regularice su publicación.

2. Existe un instrumento adecuado para el control y difusión de las tesis doctorales españolas, que es la base de datos Teseo, que podría resultar de gran utilidad, simplemente mediante el cumplimiento de la normativa por parte de todas las universidades para que sea exhaustiva y la actualización permanentemente por parte de los responsables de su mantenimiento.

3. La decisión de publicar todas las tesis doctorales, que han adoptado ya varias universidades, utilizando soportes de bajo coste como la microficha, el disquete de ordenador, el CDRom, y ya en estos momentos la edición electrónica accesible en red, pone al alcance de los usuarios los resultados de la labor investigadora de la universidad y resuelve todos los problemas que la consulta de las tesis doctorales inéditas viene planteando a diario a los usuarios y a las bibliotecas.

Los originales de las tesis entregados a la universidad, una vez finalizados los trámites administrativos, deben reunirse para ser conservados en un depósito bibliográfico único, que garantice su proceso técnico, su conservación, su difusión y el acceso a la información que contienen.

La base de datos Teseo, reúne todas las tesis doctorales leídas en universidades españolas desde 1976. Su ritmo de actualización es lento y no garantiza la exhaustividad para los trabajos posteriores a 1997. (Fuentes-González, 2002, p. 232)

Conviene advertir que la calidad de la base de datos Teseo es bastante desigual, pues depende del rigor con que los doctorandos y tribunales de tesis cumplimentan el formulario y de la diligencia con que se envíen por parte de las universidades donde las tesis son defendidas. Entre otras limitaciones presenta las siguientes: la cobertura no es ni mucho menos exhaustiva (existen omisiones), la actualización es muy lenta y los registros adolecen de carencias y errores manifiestos (Delgado et al., 2006, p. 497).

Su eficacia, (...), se ve muy limitada en la práctica, porque, al menos hasta ahora, la base de datos Teseo no es exhaustiva. (Moralejo, 2000, p. 235)

En este sentido, también hemos observado que los catálogos de las diferentes universidades 
están incompletos (...). Siendo las Tesis Doctorales el patrimonio investigador de nuestras universidades, sorprende la ausencia de rigor en la divulgación de las mismas, así como que estén relagadas a un apartado menor en las bibliotecas informatizadas de las universidades. (Martínez, 2004, p. 239)

Por ello, la solución que buscan los autores es procurar su publicación, bien en forma de monografía, mayoritariamente en el caso de las tesis de las áreas de las humanidades y de las ciencias sociales, bien en forma de artículos de revista, seleccionado aquello que considere más novedoso o de interés par la comunidad científica. Por ello frecuentemente se cuenta con dos versiones de un mismo trabajo, el englobado en lo que se llama "literatura gris", mal conocido, pero de primera mano; y la versión "comercial", en la que los criterios de exhaustividad y rapidez en dar a conocer los resultados no tienen porqué ser los prioritarios. (Miguel, 2000, p. 651)

[Existen otras fuentes nacionales e internacionales], para paliar [parte de estos obstáculos] y completar la información, (...) como la base de datos TDC@t (este proyecto se desarrolla en el ámbito de la Biblioteca Digital de Catalunya (BDG) y contiene en formato digital las tesis doctorales leídas en universidades de Cataluña). (Fuentes-González, 2002, p. 232)

O las recogidas en un artículo de Merlo y Sorli. En este artículo se ha pretendido seleccionar y comentar los principales recursos presentes en Internet para acceder bien a información bibliográfica sobre tesis doctorales, bien a los textos completos de estos documentos. Los servidores se han dividido en dos grandes grupos: recursos internacionales y recursos españoles.

[Entre los internacionales] (...) se recopilan y analizan los servidores y páginas web desde los cuales se puede obtener información de tesis doctorales leídas en distintos países. (...). [ [ La red] NDLTD [es un] proyecto piloto (...) de bibliotecas digitales de tesis y tesinas nace en 1996 con la idea de dar acceso a trabajos de investigación en formato electrónico de todo el mundo. (...). Contiene tesis independientes, enviadas directamente por los autores. Catálogo colectivo, datos en formato propio pero también en formato MARC, préstamo interbibliotecario y/o texto completo. Esta red colabora con la UNESCO, ofreciendo recursos para su ETD Guide, en la que se recopilan enlaces a proyectos sobre tesis electrónicas y otras informaciones relacionadas con el acceso en línea a trabajos académicos de investigación.
[También las siguientes:] Base de datos comercial http://wwwlib.umi.com

Dissertation.com - http://Dissertation.com: Dissertations Online -

http://www.dissertationsonline.com

CRL Dissertations Database -

http://wwwcrl.uchicago.edu/dbdissertations/sear ch.asp

Cyberthèses, publication et difussion en ligne des thèses - http://www.cybertheses.org Tesis Europeas sobre América Latina (Redial) http://www.red-redial.org

Electronic Theses and Dissertations in the Humanities -

http://etext.lib.virginia.edu/ETD/ETD.html

Doctoral dissertations in musicology-online (DDM) -

http://www.music.indiana.edu/ddm/index.html

Dissertations on Architectural History -

http://faculty.washington.edu/jn/diss.htm.

[Entre los recursos nacionales: ] (...) TESEO, principal fuente de información sobre tesis doctorales leídas en España, incluye todas las universidades. Es la más completa (formulario oficial), actualizaciones periódicas, multidisciplinar; Biblioteca Miguel de Cervantes - Catálogo de tesis, (...) incorporación por convenio o por iniciativa de los autores, aparte de la consulta automática a través del motor de búsqueda del servidor: ficha completa e información adicional (reseñas de las tesis, publicaciones del autor relacionadas, etc. (...); TDc@t , datos de la ficha de cada tesis (...); Universia - Tesis doctorales. Centro de Estudios Avanzados en Ciencias Sociales, Instituto Juan March de Estudios e Investigaciones. Tesis doctorales y estudios sobre comunicación y periodismo. (...) Muchas son las universidades españolas que ofrecen relaciones o bases de datos en las que se pueden conocer las tesis doctorales aprobadas en las mismas. (Merlo y Sorli, 2002, p. 95-106)

Diversos autores han realizado trabajos tanto para buscar y analizar los recursos existentes de acceso a las tesis doctorales, las formas de evaluarlas, como conocer los perfiles característicos de ellas, en general y en distintas especialidades, si las tesis promueven la investigación, etc. [Ver anexo I]

(...) No han sido pocos los trabajos que se han ocupado de determinar los perfiles característicos de las tesis doctorales españolas defendidas en diferentes especialidades como la Psicología, Geografía, Biblioteconomía y Documentación, Educación, Matemática, Educación Física, Ornitología, Anestesiología, Reumatología, Rehabilitación, Cuidados paliativos. (Delgado et al., 2006: 496) (...) Para conocer los perfiles característicos de las tesis se han estudiado las si- 
guientes variables: evolución del número de tesis leídas por año, centro y universidad de lectura, temática de la tesis (disciplina y unidades de análisis objeto de estudio), dirección de tesis (productividad de los directores de tesis), presencia en los tribunales de evaluación y genealogías director-doctorando. (Delgado et al., 2006, p. 497)

Determinar si la obtención de un doctorado es un factor que promueve la investigación de las carreras de los autores. (...). La mayoría de los escritores de tesis doctorales no publican los resultados en revistas internacionales. (...) Esto pone en duda la eficacia de la tesis doctoral como un factor que promueve la investigación. (Figueredo, et al., 200, p. 124-130)

Por lo general, este tipo de estudios supone un avance en el progreso científico, por lo que la difusión de los mismos es de gran interés para docentes, profesionales e investigadores. Hasta el momento, las tesis doctorales sólo se podían consultar a partir de ediciones impresas o microfilmadas, lo cual implicaba toda una serie de obstáculos para su propagación y para que los resultados obtenidos tuviesen rápida incidencia en los ámbitos científicos. Todos estos problemas se han resuelto gracias a la variedad de formatos que permiten digitalizar un documento y a los distintos sistemas de acceso telemático mediante los que se pueden conseguir dichos documentos. (Merlo y Sorli, 2002, p. 95)

El acceso a la utilización de las tesis doctorales por los investigadores está asegurada de modo amplio merced a la digitalización de las mismas o la consulta en papel en las bibliotecas universitarias. (Lopez Yepes, Fernández y Prat, 2005: 178)

\section{Reflexiones generales}

La producción científica es, sin lugar a dudas, un índice del crecimiento de las diferentes disciplinas y en muchos casos un incuestionable criterio para valorar, al menos en parte, la calidad. Es un hecho además que cualquier dato, para ser considerado científico, requiere su divulgación, con lo cual cobra cada vez mayor importancia no sólo el promover la investigación, sino la comunicación científica. En este contexto, la intención de la formación doctoral, además de fortalecer la suficiencia investigadora, es promover la difusión de los conocimientos científicos, contribuyendo con ello al avance de la disciplina o disciplinas en cuestión. De acuerdo con lo anterior, es indudable el valor que tienen las tesis doctorales dentro de la producción científica en el marco académico, pues al lado de otro tipo de trabajos constituyen lo que se denominaría la investigación académica. (Agudelo et al., 2003, p. 604-605)

Es, además, un documento indispensable para seguir el desarrollo de una ciencia, no sólo por su contenido, que es siempre original, sumamente especializado y la vanguardia de la investigación, sino también por la exhaustiva aportación bibliográfica que suele acompañar este tipo de trabajos. (Miguel, 2000, p. 645-651)

La relación entre tesis doctorales y referencias bibliográficas citadas es muy estrecha. Una tesis doctoral debe realizar una completa y ordenada revisión de la literatura, tanto conceptual como de investigación, que exponga los hallazgos más recientes, pero que a la par ofrezca una contextualización histórica de la cuestión que se indaga. (Vallejo, Fernández y Torralbo, 2006, p. 384)

Se impone, por tanto, la necesidad de establecer cambios en el proceso de evaluación referido a la evaluación previa, al examen por el tribunal y a una evaluación a posteriori que pueda reconocer el impacto real que ha causado la tesis doctoral en su correspondiente comunidad científica. Se trata de una evaluación a posteriori tendente a conocer la auténtica repercusión que opera el trabajo en el seno de la comunidad científica. Con ello se fomentaría, de un lado, la cultura de la calidad de este tipo de investigaciones y, de otro, se podría aplicar esta información para el reconocimiento de la labor de doctores y directores en la solicitud de proyectos de investigación. (Lopez Yepes, Fernández y Prat, 2005, p. 176-177)

La evaluación de la calidad de las universidades es un tema cada día más importante y necesario para establecer objetivos y saber hacia donde nos dirigimos, siendo la productividad científica de sus miembros uno de los criterios más usados en la evaluación de esta calidad. Siguiendo a Buela-Casal (2005a), a través de la productividad científica podemos conocer la situación de las universidades españolas comparándolas con otras universidades del mundo, analizar la relación entre diferentes indicadores de productividad científica (por ejemplo, tramos de investigación o programas de doctorado con mención de calidad) o la relación entre la proporción de profesores titulares de universidad y profesores catedráticos de universidad y los tramos de investigación. En ese sentido, hay una clara relación entre doctorados con mención de calidad y diferentes criterios de productividad científica, tales como la proporción de becas FPU por profesor, la proporción de proyectos I+D por profesor, porcentaje de tramos de investigación, proporción de artículos publi- 
cados en revistas indexadas en el Institute for Scientific Information (ISI) y, finalmente, la proporción de tesis doctorales por profesor (BuelaCasal, 2005b, p. 112, en Moyano, Delgado y Buela, 2006)

\section{A modo de conclusión}

Después de haber analizado exhaustivamente la bibliografía sobre las tesis doctorales, y a través de las propias palabras de los distintos autores recogidas en los apartados anteriores, podemos concluir, de forma esquemática, con seis puntos importantes, que enumeramos a continuación:

1. La tesis doctoral sirve en primer lugar para obtener un grado académico.

2. La tesis doctoral es el trabajo de investigación por excelencia (o debería serlo) y el preludio de la investigación futura.

3. La tesis doctoral acredita la suficiencia investigadora de su autor.

\section{Anexo I - Lista de las fuentes analizadas}

Agudelo, D.; Bretón-López, J.; Ortiz-Recio, G.; PovedaVera, J.; Teva, I.; Valor-Segura, I.; Vico, C. Análisis de la productividad científica de la psicología española a través de las tesis doctorales. // Psicothema. 15:4 (2003) 595-609.

Anglada, L.; Bárcena, I.; Cambras, J.; Comellas, N.; Huguet, M. Acceso electrónico a las tesis doctorales de Cataluña. // El profesional de la información. 11:1 (2002) 28-33

Anglada, L.; Reoyo, S. Els dipòsits electrònics col-lectius del CBUC: antecedents i situació actual. // Item: revista de biblioteconomia i documentació. 41 (2005) 5565.

Barbosa, A. Diez años de investigación ornitológica en España a través de las tesis doctorales. // Revista ibérica de ornitología. 47:2 (2000) 273-278.

Bastos, F.M.; Fujita Miriângela Spotti Lopes. La categorización de áreas temáticas en las bibliotecas digitales de tesis y tesinas. // Congreso del capítulo español de ISKO: La dimensión del conocimiento. VII, Barcelona, 2005. 77-90.

Bonal Zazo, J. L. La investigación universitaria sobre archivos y archivística en España a través de las tesis doctorales. // Primer Congreso Universitario de Ciencias de la Documentación: Teoría, historia y metodología de las ciencias de la documentación. 2000. 351358. Madrid: Universidad Complutense de Madrid. (http://www.ucm.es/info/multidoc/revista/num10/pagina s/pdfs/Jlbonal.pdf.)

Buela-Casal, G. An Overview of Scientific Productivity of Spanish Universities. // International Journal of Clinical and Health Psychology. 5:1 (2005) 175-190.

Buttlar, L. Information sources in library and information science doctoral research. // Library \& Information Science Research. 21:2 () 227-245.

Castillo, A.; Xifra, J. Investigación bibliométrica de las tesis doctorales españolas sobre relaciones públicas. II
4. La tesis doctoral tiene que ser un instrumento de colaboración entre las universidades y las empresas.

5. La tesis doctoral tiene que ser un indicativo de la producción e investigación científica en general, pero también para cada una de las especialidades.

La tesis doctoral tiene que permitir la evaluación de la universidad y del ámbito de especialización concreto y, por lo tanto, también del nivel de la investigación de un país o de una zona geográfica concreta.

\section{Nota del editor}

$\left(^{*}\right)$ En consonancia con los objetivos de la investigación, los autores extractan la información de las diversas fuentes, indicando omisiones y completando entre corchetes la información necesaria para una lectura ágil. Para conciliar el sistema de redacción de los autores con la facilidad de lectura, no se ha utilizado el estilo de párrafo citado y se ha preservado con ligeros cambios el sistema de cita original de los autores.

Anàlisi: quaderns de comunicació i cultura. 34 (2006) 141161.

Castillo, J.; Figueredo, E.; Villalonga, A.; Sánchez Perales, G Tesis doctorales españolas sobre anestesiología y publicaciones científicas de sus autores. // Revista española de anestesiología y reanimación. 49:3 (2002) 124-130.

Centeno, C.; Hernansanz, S.; Flores, L. A.; López-Lara, F.; Rubiales, A.; Flores, L. A. Tesis doctorales relacionadas con cuidados paliativos realizadas en la universidad española entre 1985 y 2000. // Medicina paliativa. 8: (2001), 181-189.

Civera Molla, C.; Tortosa Gil, F. Estado de la investigación psicológica en España: el grado de doctor y la investigación académica (1976-1998). // Papeles del psicólogo. 79 (2001) 42-52.

Delgado López-Cózar, E.; Torres-Salinas, D.; JiménezContreras, E.; Ruiz-Pérez, R. Análisis bibliométrico y de redes sociales aplicado a las tesis bibliométricas defendidas en España (1976-2002): temas, escuelas científicas y redes académicas. // Revista española de documentación científica. 29:4 (2006) 493-524.

Delgado López-Cózar, E. La investigación en Biblioteconomía y Documentación. Gijón: Trea, 2002.

Diego, J. I.; Prim, M. P. Análisis de las tesis doctorales en otorrinolaringología presentadas en España en el periodo 1976-2005. // Acta otorrinolaringológica española. 59:6 (2008) 292-297.

Fernández Cano, A.; Torralba, M.; Vallejo, M. Previsión y prospectiva de la producción española de tesis doctorales de pedagogía (1976-2002). // Revista de investigación educativa. $26: 1$ (2008) 191-208.

Fernández Cano, A.; Torralbo, M.; Rico, L.; Gutiérrez, P.; Maz, A. Análisis cienciométrico de las tesis doctorales españolas en educación matemática. // Revista española de documentación científica. 26:2 (2003) 162-176. 
Fernández, M. Los estudios Españoles sobre adopción y acogimiento familiar 1974-2004. // Boletín de psicología. 81 (2004) 7-31.

Figueredo, E.; Sánchez Perales. G.; Villalonga, A.; CastiIlo, J. Tesis doctorales españolas sobre anestesiología y publicaciones científicas de sus autores. // Revista española de anestesiología y reanimación. 49:3 (2002) 124-130.

Fluxà, J. M. Relación entre documentación e investigación en la universidad y en la empresa. // V Jornadas Españolas de Documentación automatizada. Cáceres, 1996. 895-901.

Fuentes Pujol, M. E.; González Quesada, A. Tesis doctorales en España en información y documentación. // Morán Suárez, M. A.; Rodríguez López, M. (ed.) La Documentación para la investigación: homenaje a José Antonio Martín Fuertes. León: Universidad de León, 2002. 229-241.

Fuentes Pujol, M. E.; González Quesada, A. La investigación y la literatura especializada en Información y Documentación: una revisión. // Scire. Representación y Organización del Conocimiento. 7:2 (2001) 11-38, 14.

Fuentes Pujol, M. E. Documentación científica e información. Metodología del trabajo intelectual y científico. Barcelona: ESRP-PPU, 1992.

Fuentes Pujol, M. E.; Arguimbau, Vivó, LI. Las tesis doctorales en España (1998-2008): Estudios, estadísticas y repositorios cooperativos. 2009 (En revisión).

Gete Benavente, P. La historia de la farmacia en la España del siglo XIX a través de las tesis doctorales que tratan del tema y que se conservan en el Archivo de la Universidad Complutense de Madrid. Tesis doctoral, Universidad de Alcalá de Henares, 2005.

Gómez Sancho, José María; Mancebón Torrubia, María Jesús. Algunas reflexiones metodológicas sobre la evaluación de la eficiencia productiva de las instituciones de educación superior. // Ekonomiaz. 60 (2005) 140-165.

Guisan, C.; Expósito, P. Fifty years of Econometrics Research in Spain: Doctoral Dissertations, international publications and other contributions, 1956-2006. http://ideas.repec.org/p/eaa/ecodev/98.html. (15 enero 2009).

Hernández Borge, J. Cuarenta años de tesis doctorales en geografía en la Universidad de Santiago de Compostela (1959-1998). // Estudios geográficos. 60:237 (1999) 727-740.

Lázaro Torres, M. L. La geografía a las puertas del tercer milenio a partir de las tesis doctorales leídas en los noventa. // Estudios geográficos. 63:246 (2002) 154168.

López Huertas, M. J. La investigación española en organización del conocimiento. // Coloquio internacional de Ciencias de la Documentación. IV. Salamanca, 2003; Congreso del capítulo español de

LópeZKYepes, J. Focos de investigación y escuelas científicas en documentación: la experiencia de las tesis doctorales. // El profesional de la información. 11:1 (2002) 46-51.

López Yepes, J. Focos de investigación y escuelas científicas en documentación a través de la realización y dirección de tesis doctorales: el caso del Departamento de Biblioteconomía y Documentación de la Universidad Complutense de Madrid (1983-2001). // Documentación de las ciencias de la información. 25 (2002) 1954.

López Yepes, J.; Fernández Bajón, M. T.; Prat Sedeño, J. La investigación española en documentación informativa a examen en Ibersid 2004. Estado de la cuestión con especial referencia a las tesis doctorales (1976-2004). // El profesional de la información. 14:1 (2005) 50-57.

López Yepes, J.; Fernández Bajón, M. T.; Prat Sedeño, J. Las tesis doctorales en biblioteconomía y documentación: diagnóstico y propuesta de criterios de evaluación. // Documentación de las ciencias de la información. 28 (2005) 173-187.

López Yepes, J. La aventura de la investigación científica. Guía del investigador y del director de investigación. Madrid: Síntesis, 1995.

Magriñá Contreras, M. La difusión de la producción científica española a través del proyecto DIALNET. // Boletín de la ANABAD. 57:4 (2007) 9-22.

Martínez Cerverón, R. Maestros y discípulos en la psicología contemporánea: un estudio a través de las tesis doctorales. Tesis doctoral, Universidad de Valencia, 2007.

Martínez Pestaña, M. J. La producción de tesis doctorales sobre temas publicitarios (1971-2001). // Documentación de las ciencias de la información. 27 (2004) 237-267.

Melero, R.; Lopez Medina, A.; Prats, J. Landscape of Open Access Institutional Repositories in Spain. // Third International Conference on Open Repositories 2008, 1-4 April 2008, Southampton, United Kingdom. http://pubs.or08. ecs.soton.ac.uk/56/ (15 enero 2009).

Miguel Alonso, A. Aportaciones al estudio de la literatura gris universitaria: La evolución de la tesis doctoral en España. Primer Congreso Universitario de Ciencias de la Documentación: Teoría, historia y metodología de las ciencias de la documentación. 2000. p. 645-651. Madrid: Universidad Complutense de Madrid.

Moralejo Álvarez, M. R. Las tesis doctorales de las universidades españolas: control bibliográfico y acceso. // Revista general de información y documentación. 10:1 (2000) 235-243.

Moyano, M.; Delgado Domínguez, C.; Buela Casal, G. Análisis de la productividad científica de la Psiquiatría española a través de las tesis doctorales en la base de datos TESEO (1993-2002). // International Journal of Psychology and Psychological Therapy. 6:1 (2006) 111-120.

Moyle, M. Improving Access to European E-theses: the DARTEurope Programme. // LIBER Quarterly. 18:3/4 (2008) 413-423.

Muñoz Muñoz, A. M. The Scholarly Transition of Female Academics at the University of Granada (1975-1990). // Scientometrics. 64:3 (2005) 325-350.

Muñoz Muñoz A. M. Tesis doctorales defendidas por las profesoras de la Universidad de Granada (1975-1990). // Revista de Documentación Científica. 27:4 (2004) 499-505.

Muñoz Tinoco, C.; Peña Arrebola, A. Tesis doctorales sobre rehabilitación presentadas en España en el periodo 19761996. // Rehabilitación. 32:4 (1998) 221-4.

Muñoz Tinoco, C.; Peña Arrebola, A. Tesis doctorales sobre reumatología presentadas en España entre 1976 y 1997. Estudio bibliométrico. // Revista española de reumatología. 3:27 (2000) 93-98.

Nagore Ferrer, M. Tesis doctorales. La investigación musical en España: situación actual y perspectivas de futuro. // Revista de musicología. 28:2 (2005) 1451-1470.

Orera Orera, L. Bibliotecas digitales de tesis doctorales: Metodología para su planificación. // Boletín de la Asociación Andaluza de Bibliotecarios.72 (2003) 55-72.

Orera Orera, L. La edición digital de tesis doctorales: hacia la resolución de los problemas de accesibilidad. // Revista interamericana de bibliotecología. 26:1 (2003) 11-35.

Orera Orera, L. Las tesis sobre biblioteconomía y bibliotecas y su accesibilidad a través de las bibliotecas universitarias españolas. // Fernández Bajón, M. T.; López López, P.; López Yepes, J. (ed.). Estudios de biblioteconomía y do- 
cumentación. Homenaje a la profesora María Rosa Garrido Arilla. Madrid, Universidad Complutense de Madrid, 2004. 91-102.

Ortega Martínez, E.; Rodríguez Herráez, B. La investigación turística a través de tesis doctorales. Un análisis comparativo entre España y Francia. // Estudios turísticos. 159 (2004) 5-27.

Pablo Núñez, L. Aproximación bibliográfica a los estudios de semántica estructural en España. // Hesperia: anuario de filología hispánica. 9 (2006) 163-181.

Pérez López, I. Historia antigua y ciencias afines en las publicaciones periódicas y tesis doctorales del último tercio del siglo XIX español (1868-1900). Tesis doctoral, Universidad de Málaga, 2007.

Ponce De León Elizondo, A.; Gargallo Ibort, E.; Loza Olave, E. Análisis de las tendencias en las tesis doctorales de educación física. Cursos 1980-1981 a 19951996. // Apunts. 52 (1998) 104-108.

Rorralbo Rodríguez, M. Análisis cientimétrico, conceptual y metodológico de las tesis doctorales españolas en educación matemática 1976-1998. Tesis doctoral, Universidad de Granada, 2001.

Sabater Lorenzo, P. Las tesis doctorales de las facultades de ciencias de la Universidad de Murcia, 1955-1990: catálogo, estadística descriptiva y bibliometría. Tesis doctoral, Universidad de Murcia, 2001.

Sierra Bravo, R. Tesis Doctorales y trabajos de Investigación científica. $3^{a}$. ed. Rev y Ampl. Madrid: Paraninfo, 1993.

Spála, M; Bratková, E. [University qualifying work (dissertations), their current registration, electronic access and related technical, administrative and legal questions] .// Cas Lek Cesk. 143:3 (2004) 202-4.

Sorli Rojo, A.; Merlo Vega, J. A. Base de datos y recursos en Internet de tesis doctorales. // Revista de documentación científica. 25:1 (2002) 95-106.

Torres Ramírez, I.; Torres Salinas, D. Tesis doctorales sobre estudios de las mujeres en España (19762002). A propósito de un indicador definitivo en investigación. // Revista española de documentación científica. $28: 4$ (2005) 479-499.
Urbano, C. El análisis de citas en publicaciones de usuarios de bibliotecas universitarias: estudio de las tesis doctorales en informática de la Universidad Politécnica de Cataluña, 1996-1998. Tesis doctoral, Universidad de Barcelona, 2000.

Urbano, C. Tipologia documental citada en tesis doctorals d'informàtica: bases empíriques per a la gestió equilibrada de colleccions. // BiD: textos universitaris de biblioteconomia i documentació, 2000, núm. 5. http://www.ub.edu/ biblio/bid/05urban1.htm. (15 enero 2009)

Vallejo Ruiz, M.; Fernández Cano, A.; Torralbo Rodríguez, M. Patrones de citación en la investigación española en educación matemática. // Revista española de documentación científica. 29:3 (2006) 382-397. http://Redc.revistas. csic.es/index.php/article/view/295/366.

Vallmitjana, N.; Sabaté, L. G. Citation Analysis of Ph.D. Dissertation References as a Tool for Collection Management in an Academic Chemistry Library. // College \& Research Libraries. 69:1 (2008) 72-81.

Vega, J. A.; Sorli Rojo, A. Bases de datos y recursos en Internet sobre tesis doctorales. // Revista española de documentación científica. 25:1 (2002) 95-106.

Villarroya, A. PhD Theses in Spain: a Gender Study covering the Years 1990-2004. I/ Scientometrics. 77:3 (2008) 469483.

Zapico Alonso, F. La investigación en documentación: análisis bibliométrico de las tesis en Documentación. Tesis doctorales en España en información y documentación. // Morán Suárez, M. A.; Rodríguez López, M. (ed.). La Documentación para la investigación: homenaje a José Antonio Martín Fuertes. León: Universidad de León, 2002. 689-713.

Zapico, F.; Reyes Barragan, M. ; Guerreo Bote, V.; López Pujalte, C. La investigación en Documentación: análisis bibliométrico de las tesis de Documentación. // La documentación para la investigación: homenaje a José Antonio Martin Fuertes. León: Universidad de León, Secret. Publicaciones y Medios Audiovisuales, 2002, 2:689-713.

Xifra, J. Public Relations Review. 32:3 (Sep 2006) 302-308. 
Anexo II - Definiciones de investigación científica (López Yepes, 1995: 40-41)

\begin{tabular}{|ll|}
\hline Roger Rivière (1969) & $\begin{array}{l}\text { Investigar es profundizar, desarrollar, buscar nue- } \\
\text { vas adquisiciones. }\end{array}$ \\
Rodríguez Villanueva (1986) & $\begin{array}{l}\text { La investigación científica puede ser definida de } \\
\text { forma simple y clara como la búsqueda de infor- } \\
\text { mación, de la verdad y de lo desconocido. En rea- } \\
\text { lidad, es una lucha por expandir el conocimiento. }\end{array}$ \\
Paniker & $\begin{array}{l}\text { Investigar es mererse a seguir los vestigios que algo } \\
\text { existente, real, ha dejado a su paso. La investiga- } \\
\text { ción es la búsqueda de lo que es, de la esencia de }\end{array}$ \\
las cosas a partir de sus huellas, de sus rastros... \\
Bunge (1969) \\
Proceso de interrogación a cuestiones no conoci- \\
das por medio de determinados procedimientos \\
que, en su conjunto, constituyen el método. \\
La investigación científica arranca con la percep- \\
ción de que el acervo de conocimientos disponi- \\
bles es insuficiente para mancjar determinados \\
problemas. No cmpieza con un borrón y cuenta \\
nueva, porque la investigación se ocupa de proble- \\
mas, y no es posible formular una pregunta -por \\
no hablar ya de darle respuesta- fuera de algún \\
cuerpo de conocimiento: sólo quienes ven pueden \\
darse cuenta de que falta algo.
\end{tabular}

\section{Anexo III - Nueva normativa de doctorado}

La adaptación al EEES se asienta sobre los fundamentos jurídicos establecidos por el RD 1393/2007, 29 de octubre (BOE 260, 30 de Octubre 2007) promulgado con el objetivo de adaptar los estudios españoles de postgrado a las nuevas directrices europeas. Entre otras innovaciones, cabe destacar la posibilidad de obtener la mención de doctor europeo o la presentación de tesis doctorales como compendio de publicaciones. En el modelo anterior (reglamentado por el RD 778/1998) los estudios de doctorado incorporaban una parte de docencia y otra de investigación, mientras que el nuevo modelo parece orientarse más decididamente hacia las tareas de formación y desarrollo científico, que culminan en la tesis doctoral, definida como un trabajo original de investigación.

El artículo 11 del RD 1393/2007, en sus puntos 1 y 4 se expone la filosofía general del Doctorado: 1. Las enseñanzas de Doctorado tienen como finalidad la formación avanzada del estudiante en las técnicas de investigación, podrán incorporar cursos, seminarios u otras actividades orientadas a la formación investigadora e incluirá la elaboración y presentación de la correspondientes tesis doctoral, consistente en un trabajo original de investigación. (...) 4. Así mismo, de acuerdo con lo que establezca la normativa sobre expedición de títulos, se incluirá información que especifique la disciplina en la que se ha elaborado la Tesis Doctoral.
En el mismo Real Decreto (RD) 1393/2007, pero en su artículo 22 puntos 1 y 2 explica como puede obtenerse esta mención de doctor europeo:

1. Se podrá incluir en el anverso del título de Doctor o Doctora la mención «Doctor europeo», siempre que concurran las siguientes circunstancias: a) Que, durante el periodo de formación necesario para la obtención del título de doctor, el doctorando haya realizado una estancia mínima de tres meses fuera de España en una institución de enseñanza superior o centro de investigación de un Estado miembro de la Unión Europea, cursando estudios o realizando trabajos de investigación que le hayan sido reconocidos por la universidad; b) Que parte de la tesis doctoral, al menos el resumen y las conclusiones; se haya redactado y sea presentado en una de las lenguas oficiales de la Unión Europea distinta a cualquiera de las lenguas oficiales en España; c) Que la tesis haya sido informada por un mínimo de dos expertos pertenecientes a alguna institución de educación superior o instituto de investigación de un Estado miembro de la Unión Europea distinto de España; d) Que, al menos, un experto perteneciente a alguna institución de educación superior o centro de investigación de un Estado miembro de la Unión Europea distinto de España, con el título de doctor, y distinto del responsable de la estancia mencionada en el apartado a) y los mencionados en el apartado c), haya formado parte del tribunal evaluador de la tesis. 2. La defensa de la tesis ha de ser efectuada en la propia Universidad en la que el doctorando estuviera inscrito. 